\title{
Leukotriene B4 in inflammatory bowel disease
}

\author{
WILLIAM F STENSON, MD
}

WF STENSON. Leukotriene $\mathrm{B}_{4}$ in inflammatory bowel disease. Can J Gastroenterol 1993;7(2):182-186. Leukotriene $B_{4}$ is an inflammatory mediator produced by metabolism of arachidonic acid through the 5-lipoxygenase pathway in neutrophils, macrophages and mast cells. The major biological effect of leukotriene $\mathrm{B}_{4}$ is the activation of circulating neutrophils to bind to the vascular endothelium and migrate into inflamed tissues. There are markedly elevated levels of leukotriene $\mathrm{B}_{4}$ in inflammatory bowel disease and there is considerable evidence that it is an important neutrophil activator in this disease. Preliminary studies suggest that inhibition of leukotriene $B_{4}$ synthesis may be therapeutic in inflammatory bowel disease.

Key Words: 5-Lipoxygenase, Arachidonic acid, Leukotriene B4, Neutrophil, Ulcerative colitis

\section{Leucotriène $\mathrm{B}_{4}$ dans la maladie intestinale inflammatoire}

RÉSUMÉ: Le leucotriène $\mathrm{B}_{4}$ est un médiateur de l'inflammation produit par le métabolisme de l'acide arachidonique par le biais de la 5-lipoxygénase dans les neutrophiles, les macrophages et les mastocytes. Le principal effet biologique du leucotriène $\mathrm{B}_{4}$ est l'activation des neutrophiles circulants qui se fixent à l'endothélium vasculaire et migrent vers les tissus inflammés. Les taux de leucotriène $\mathrm{B}_{4}$ sont nettement élevés dans la maladie intestinale inflammatoire et les résultats démontrent qu'il est un important activateur des neutrophiles dans cette maladie. Les études préliminaires suggèrent que l'inhibition de la synthèse du leucotriène B4 puisse être thérapeutique dans la maladie intestinale inflammatoire.

Department of Medicine, Jewish Hospital of St Louis, St Louis, Missouri, USA

Correspondence and reprints: Dr William F Stenson, Dept. of Medicine, Jewish Hospital of St Louis, St Louis, MO 63110, USA. Telephone (314) 362-8951, Fax (314) 362-8959
$\mathrm{T}$ HE PATHOGENESIS OF INTESTINAL inflammation in general, and in. flammatory bowel disease (IBD) in particular, can be divided into two stages The first stage is the initiating event which triggers the inflammatory response, but in IBD the initiating event is unknown. The second stage is the amplification of the inflammatory response which involves a number of inflammatory cells including lymphocytes, mast cells, macrophages and neutrophils. The amplification of the inflammatory response is controlled by soluble mediators including histamine, serotonin, products of the complement pathway, prostaglandins and leukotrienes. This second stage, the amplification of the inflammatory response, is important in the pathogenesis of IBD for two reasons. First, it is the amplification of the inflammatory response, and not the initiating event, which is the cause of the tissue destruction and the histologic and functional changes characteristic of IBD. Second, those druge which are effective in the treatment of IBD appear to exert their therapeutic 
effects by modulating the production of soluble mediators $(1,2)$. Moreover, as long as the initiating event remains unknown, it is likely that further advances in the medical therapy of IBD will result from pharmacologic modulation of inflammatory mediators.

\section{PROSTAGLANDINS IN IBD}

Few investigations have focused directly on mediators of the inflammatory process in IBD. Prostaglandins, which are found in increased concentrations in inflammatory exudates, have received the most attention $(3,4)$. High levels of prostaglandins are present in rectal mucosa and in serum in IBD and high levels of prostaglandin metabolites are found in the urine. Prostaglandin levels decline when patients with IBD are treated with either corticosteroids or sulphasalazine. However, prostaglandins also decline when IBD patients are treated with nonsteroidal anti-inflammatory drugs (eg, indomethacin), but patients do not show clinical improvement. There is, in fact, some evidence that nonsteroidal agents may increase the severity of IBD. These last findings suggest that prostaglandins may not be important mediators in IBD and that the mechanism of action of corticosteroids and sulphasalazine may not relate to the inhibition of prostaglandin synthesis.

\section{LIPOXYGENASE PRODUCTS IN IBD}

More recently some work has been directed at the potential role of lipoxygenase products in the pathogenesis of $\operatorname{IBD}(3,5)$. Incubation of IBD mucosa from surgical resections with exogenous arachidonic acid leads to the synthesis of both lipoxygenase products (leukotriene $\mathrm{B}_{4}$ [LTB4], 5 . hydroxy-6,8,11,14-eicosatetraenoic acid [5-HETE], 12-hydroxy-5,8,10,14eicosatetraenoic acid [12-HETE] and 15-hydroxy-5,8,11,13-eicosatetraenoic acid [15-HETE]), and cyclooxygenase products (prostaglandin $\mathrm{E}_{2}\left[\mathrm{PGE}_{2}\right]$ and thromboxane $\left.B_{2}\left[T_{x} B_{2}\right]\right)(6,7)$. In comparison, arachidonate incubated with normal colonic mucosa is esterified into phospholipids and triglycerides, but is not metabolized by lipoxygenase or cyclooxygenase. The metabolism of arachidonate by IBD mucosa is remarkable in that the exogenous arachidonate is metabolized by lipoxygenase and cyclooxygenase even in the absence of added stimuli. This is in contrast to neutrophils, for example, in which exogenous arachidonate is metabolized only in the presence of a stimulus.

In order to determine whether or not these lipoxygenase products exist in the tissue endogenously, lipids were extracted from the colonic mucosa and separated by high performance liquid chromatography (HPLC). LTB4, 12 HETE, 15-HETE and 5-HETE were present endogenously in the IBD mucosa. Whereas normal mucosa contains less than $5 \mathrm{ng} \mathrm{LTB} 4 / \mathrm{g}$ mucosa (which was the lower limit of sensitivity of the assay), the average LTB4 content of IBD mucosa was $254 \mathrm{ng} / \mathrm{g}$ mucosa (7). If this concentration of $\mathrm{LTB}_{4}$ was in solution, it would be well within the biologically active range. In addition, incubation of arachidonate with rectal mucosal biopsies from IBD patients results in the synthesis of the sulphidoleukotrienes (LTC4, LTD4, $\mathrm{LTE}_{4}$ ) in addition to $\mathrm{LTB}_{4}(8)$.

There is also in vivo evidence for the enhanced production of $\mathrm{LTB}_{4}$ in ulcerative colitis (9). Bags of dialysis tubing were placed in the rectums of patients with ulcerative colitis and left in place for $4 \mathrm{~h}$. The dialysate was assayed for $\mathrm{PGE}_{2}$ and $\mathrm{LTB}_{4}$. The levels of $\mathrm{PGE}_{2}$ and $\mathrm{LTB}_{4}$ were considerably higher in ulcerative colitis patients than in normals. Moreover, the $\mathrm{PGE}_{2}$ and $\mathrm{LTB}_{4}$ concentrations in the dialysates correlated with the severity of ulcerative colitis as assessed histologically, endoscopically and clinically. Treatment with prednisolone enemas for two to four weeks resulted in clinical improvement and a decline in $\mathrm{PGE}_{2}$ and $\mathrm{LTB}_{4}$ levels.

Studies on the profile of eicosanoids in the inflamed rectum have demonstrated that orally administered prednisolone decreases concentrations of both $\mathrm{PGE}_{2}$ and $\mathrm{LTB}_{4}$ in rectal dialysates, whereas systemic indomethacin reduces $\mathrm{PGE}_{2}$ concentra- tions in the face of unchanged, or even increased, LTB4 levels (10). Prednisolone is therapeutic in IBD whereas indomethacin is not. A recent study compared levels of $\mathrm{PGE}_{2}, \mathrm{PGF}_{2} \alpha$ thromboxane $\mathrm{B}_{2}$, 6-keto $\mathrm{PGF}_{1 \alpha}$ and $\mathrm{LTB}_{4}$ in rectal dialysates from controls, ulcerative colitis, Crohn's disease and Clostridium difficile-associated colitis (11). All patients had endoscopically proven rectal inflammation. Concentrations of $\mathrm{PGE}_{2}, \mathrm{PGF}_{2 \alpha}$ and thromboxane $\mathrm{B}_{2}$, but not 6-keto $\mathrm{PGF}_{1 \alpha}$, were increased in all disease groups compared with controls. Concentrations of LTB4 were substantially increased in ulcerative colitis, but in Crohn's disease and C difficile-associated colitis only those patients with rectal ulcers showed elevations of $\mathrm{LTB}_{4}$. Thus, the role of $\mathrm{LTB}_{4}$ in the pathogenesis of ulcerative colitis may be greater than its role in Crohn's disease.

\section{ARACHIDONATE METABO- LISM IN ANIMAL MODELS OF INTESTINAL INFLAMMATION}

There are no totally satisfactory animal models of human IBD; however studies of eicosanoid production in three animal models of intestinal inflammation revealed striking similarities to that seen in IBD. Zipser measured PGE $2 \mathrm{LTB}_{4}$ and $\mathrm{LTC}_{4}$ levels by rectal dialysis in rabbits in whom colitis had been induced with immune complexes (12). Colitis was associated with enhanced synthesis of all three compounds, $\mathrm{LTB}_{4}>\mathrm{PGE}_{2}>\mathrm{LTC}_{4}$. There was good correlation between elevated levels of $\mathrm{LTB}_{4}$ and presence of histologic inflammation and diarrhea production. Arachidonate metabolism was also assayed in a second model of intestinal inflammation - acetic acid colitis in the rat.

Dilute acetic acid was injected into rat colon and effects were observed after $24 \mathrm{~h}$ (13). Histologic analysis of this model of intestinal inflammation showed the formation of ulcers and profound neutrophil infiltration. Arachidonic acid metabolism in colonic mucosa from acetic acid treated rats was compared with that from normal rats. The normal rat mucosa meta- 
bolized only a very small portion of the exogenous arachidonic acid, whereas the colonic mucosa from acetic acid treated rats converted a significant portion of exogenous arachidonate to lipoxygenase products - $\mathrm{LTB}_{4}, 5$ HETE, 12-HETE and 15-HETE. Moreover, when the endogenous mucosal lipids of the normal and acetic acid treated rats were compared, the acetic acid treated mucosa was found to have significant amounts of $\mathrm{LTB}_{4}, 5$ HETE, 12-HETE and 15-HETE. These compounds were not present in the normal mucosa. Thus, arachidonic acid metabolism in the acetic acid treated mucosa closely resembles that in human IBD.

Although IBD is viewed as a chronic inflammatory process, histologically and functionally it is to some extent a prolonged acute inflammatory response. The acuteness of the inflammatory response correlates with the presence of numerous neutrophils in the mucosa. The pattern of arachidonic acid metabolism in acetic acid colitis closely resembles that of stimulated peripheral blood neutrophils with $\mathrm{LTB}_{4}$ and 5-HETE being the most prominent products. An experiment was performed with neutrophil depleted rats (14) to determine if the neutrophils that infiltrate the mucosa in acetic acid colitis were an important source of arachidonate metabolites. Rats were treated with antineutrophil serum raised in rabbits. The antineutrophil serum caused a fall in the blood neutrophil count from (mean \pm $\mathrm{sd}, \mathrm{n}=4) 2493 \pm 464$ neutrophils $/ \mathrm{mm}^{3}$ to $652 \pm 884$ neutrophils $/ \mathrm{mm}^{3}$. The neutrophil depleted rats were then treated with acetic acid. Twenty-four hours later the rats were killed and the colonic mucosa was incubated with arachidonic acid and the ionophore A23187. The mucosa from normal rats produced $\mathrm{LTB}_{4}$ and 5 -HETE in addition to 12-HETE, 11-HETE and 15. HETE. The mucosa from the neutrophil depleted rats produced similar amounts of 12-HETE, 11-HETE and 15-HETE, but much less LTB4 or 5-HETE (each reduced by $85 \%$ ), suggesting that in acetic acid colitis mucosa the major source of $\mathrm{LTB}_{4}$ and
5-HETE is the neutrophils that infiltrate the mucosa.

There are two points to be taken from the studies of arachidonate metabolism in IBD and acetic acid colitis. First, the arachidonate metabolites formed in IBD mucosa are formed primarily by components of the acute rather than the chronic portion of the inflammatory response. The most important cellular component appears to be the neutrophil. Second, the pattern of arachidonate metabolism seen in IBD mucosa is not specific to IBD and is probably common to all forms of intestinal inflammation with an acute component.

In the third model of colitis, inflammation is induced by administration of trinitrobenzenesulphonic acid (TNBS). Rectal administration of a single dose of TNBS in rats induces acute colitis followed by chronic colitis and then healing with stricture formation. Three weeks after administration of TNBS, at a time when histologic assessment revealed chronic inflammation, macroscopic colonic damage correlated with an increase in synthesis of 6-keto PGFl $\alpha$ and $\mathrm{LTB}_{4}$ (15). Daily intracolonic treatment with a specific 5 . lipoxygenase inhibitor, L651,392, during the first four days after initiation of the colitis with TNBS resulted in significant reductions of LTB 4 synthesis and colonic damage score (16). When examined two weeks after initiation of colitis the group treated with L651,392 (for the first four days) showed significantly less colonic damage (assessed macroscopically and histologically) and colonic inflammation (assessed histologically and by measurement of myeloperoxidase activity). Using the same TNBS model of colitis another group studied the effect of treatment with 16,16'-dimethlyprostaglandin $\mathrm{E}_{2}$, a cytoprotective compound (17). Animals treated with 16,16'-dimethyl PGE 2 had decreased inflammation (assessed by gross inspection and measurement of myelo. peroxidase) and decreased LTB4 synthesis. The treated rats also developed fewer structures.

Thus, treatment of an animal model of colitis with a specific 5-lipoxygenase inhibition L651,392 or with a cytoprotective prostaglandin can reduce inflammation. It can be conjectured that L651, 392 reduces inflammation by reducing $\mathrm{LTB}_{4}$ production, whereas, 16,16'-dimethyl PGE? reduces inflammation by blocking the mucosal damage induced by TNBS. In the second case there is less LTB4 produced because there are fewer inflammatory cells which are capable of synthesizing $\mathrm{LTB}_{4}$.

\section{LIPOXYGENASE PRODUCTS AND BIOLOGICAL FUNCTION IN IBD}

It is easier to demonstrate the presence of lipoxygenase products in IBD mucosa than to define precisely their role in the pathogenesis of the inflammatory response in IBD. Among the mediators which may contribute to the inflammatory response in IBD are products of the complement cascade, kinins, prostaglandins, histamine and platelet activating factor. These mediators have similar biologic proper. ties and it is difficult to sort out the contribution of each to a particular inflammatory event.

There are several functional and his. tologic changes seen in IBD that may be products of the enhanced synthesis of lipoxygenase products. One of the hallmarks of intestinal inflammation in general and IBD in particular is mucosal edema. The mucosa is thick. ened and, on endoscopy, the vascular pattern is obscured. Among the soluble mediators of inflammation that enhance vascular permeability are histamine, C5a, bradykinin and LTB4. It is now clear that there is differential susceptibility to enhance vascular permeability in different organs. Injection of $\mathrm{LTB}_{4}(3 \mu \mathrm{g} / \mathrm{kg})$ into the left ventricle of the rat induced enhanced vasculat permeability in the skin, the aorta, the stomach, the small bowel and the cecum (18). The combination of LTB and $\mathrm{PGE}_{2}$ was more potent than LTB, alone. In contrast, neither $\mathrm{PGE}_{2}$ not $\mathrm{LTB}_{4}$ had any effect on vascular permeability in the brain, heart and testes Thus, LTB4 may contribute to enhanced vascular permeability in IBD.

Diarrhea is a prominent clinical fea- 
ture of IBD and probably includes elements of both impaired absorption and enhanced secretion. The effects of lipoxygenase products on salt and water flux in intestinal epithelium have not been fully explored. However, the first intermediate in the lipoxygenase pathway, 5-HPETE, has been shown to enhance chloride secretion in the rabbit colon in vitro (19).

Although they are considered to be chronic inflammatory diseases the histologic picture of both Crohn's disease and ulcerative colitis includes intense neutrophil infiltration. In vivo studies with radiolabelled peripheral blood neutrophils demonstrate the movement of large numbers of neutrophils out of the circulation into the inflamed mucosa and the intestinal lumen (20). This process suggests the presence of a potent chemotactic agent in the intestinal mucosa which promotes the passage of infiltrating neutrophils between endothelial cells, through the basement membrane and into the intestinal tissue.

Among the soluble mediators of inflammation that are important neutrophil chemotactic agents are: C5a, which is part of the complement cascade; bacterial-derived peptides including formylmethionlleucylphenylalanine; and the arachidonate metabolites LTB4 (21) and 5-HETE. To determine which, if any, of these compounds was the mediator of neutrophil infiltration of the mucosa in IBD a study was performed using a Boyden chamber with $51 \mathrm{Cr}$-labelled neutrophils in the upper chamber and either a chemoattractant or buffer in the lower chamber (22). Two filters, one on top of the other, separated the two chambers. After a $3 \mathrm{~h}$ incubation period, the amount of radioactivity present in each filter was determined and the results expressed as the percent of total radioactivity present in the lower of the two filters. When buffer alone was present in the lower chamber almost none of the neutrophils penetrated the upper filter and entered the lower filter. When a chemotactic agent was present in the lower chamber a significant portion of the cells penetrated the upper filter and entered the lower. A dose-response curve was obtained when various amounts of synthetic $\mathrm{LTB}_{4}$ were placed in the lower chamber. Next, homogenates of human colonic mucosa were added to the lower chamber at concentrations of $2 \mathrm{mg} / \mathrm{mL}, 20 \mathrm{mg} / \mathrm{mL}$ and $100 \mathrm{mg} / \mathrm{mL}$. The chemotactic response to ulcerative colitis mucosa was more than 20 times that to normal mucosa and the response to Crohn's colitis mucosa was more than 10 times that to normal mucosa.

To characterize the nature of the chemotactic agent in ulcerative colitis mucosa the chemotactic activity in homogenates of ulcerative colitis mucosa was compared to that in the lipid extracts of the homogenates. Results showed chemotactic activity in the lipid extract to be between $78 \%$ and $90 \%$ of the chemotactic activity in the total homogenate, suggesting that much of the chemotactic activity was present as a lipid. Fractions obtained by HPLC separation of the lipid extracts from ulcerative colitis mucosa were utilized in the chemotaxis assay. Only the fraction that co-eluted with $\mathrm{LTB}_{4}$ contained a significant amount of chemotactic activity, indicating that $\mathrm{LTB}_{4}$ was the predominant chemotactic agent in ulcerative colitis mucosal extracts.

\section{DRUG THERAPY OF IBD AND THE LIPOXYGENASE PATHWAY}

The two most widely used medical therapies for IBD are corticosteroids and sulphasalazine. Each of them block the synthesis of lipoxygenase products in vitro, but each also has other pharmacologic properties unrelated to arachidonate metabolism which may contribute to their therapeutic efficacy. Corticosteroids induce the synthesis of lipomodulin which blocks phospholipase A2 and thus limits the availability of arachidonate, the substrate for the cyclooxygenase and lipoxygenase pathways (1).

Sulphasalazine is metabolized to 5 ASA and sulphapyridine. While it is thought that sulphapyridine is responsible for the undesirable side effects of sulphasalazine and that 5-ASA is the therapeutic agent, there is substantial evidence that the parent compound, sulphasalazine, possesses pharmacologic properties distinct from those of 5-ASA. Both sulphasalazine and 5 ASA inhibit cyclooxygenase and 5 . lipoxygenase. In addition, sulphasalazine inhibits thromboxane synthetase. One of the difficulties in determining therapeutically relevant pharmacologic effects is determining the appropriate concentrations of these compounds for study. In treated patients the concentrations of these compounds in stool are enormous, ie, 1 $\mathrm{mm}$ for sulphasalazine and $10 \mathrm{mM}$ for 5-ASA (23). However, they are poorly absorbed and the serum concentrations are quite low. Thus, high concentrations of these agents are observed on the lumenal side of the inflamed mucosa while, at the same time, concentrations in the capillaries are minimal. The concentration of drugs to which relevant cells in the mucosa are exposed is unclear.

When tested in in vitro assay systems at concentrations found in the colonic lumen these compounds exert many pharmacologic effects, including inhibition of arachidonic metabolism; however, when tested at concentrations found in the serum, their pharmacologic effects are relatively minimal. For example, sulphasalazine inhibits a number of steps in both the cyclooxygenase pathway and the lipoxygenase pathway at a concentration of approximately $1 \mathrm{mM}$, which is the concentration found in the colonic lumen but not in the bloodstream. However, both corticosteroids and sulphasalazine have other pharmacologic properties unrelated to arachidonate metabolism which may contribute to their therapeutic efficacy in IBD. Corticosteroids affect lymphocyte proliferation whereas sulphasalazine blocks lymphocyte cytotoxicity (24). Either of these effects could contribute to their therapeutic efficacy in IBD.

ACKNOWLEDGEMENT: This work was supported by NIH grant AM33165 and a grant from the National Foundation for Ileitis and Colitis. 


\section{REFERENCES}

1. Flower RJ, Blackwell GJ. Anti-inflammatory steroids induce the biosynthesis of phospholipase A2 inhibitor which prevents prostaglandin generation. Nature 1979;278:456-9.

2. Allgayer H, Stenson WF. A comparison of effects of sulphasalazine and its metabolites on the metabolism of endogenous vs. exogenous arachidonic acid. Immunopharm. 1988;15:39-46.

3. Rampton DS, Hawkey CJ. Prostaglandins and ulcerative colitis. Gut 1985;25:1399-413.

4. Sharon P, Ligumsky M, Rachmilewitz D, Zor U. Role of prostaglandins in ulcerative colitis. Enhanced production during active disease and inhibition by sulphasalazine. Gastroenterology 1978;75:638-40.

5. Donowitz M. Arachidonic acid metabolites and their role in inflammatory bowel disease. An update requiring addition of a pathway. Gastroenterology 1985;88:5800-11.

6. Boughton-Smith NK, Hawkey CJ, Whittle BJR: Biosynthesis of lipoxygenase and cyclooxygenase products from ${ }^{14} \mathrm{C}$-arachidonic acid by human colonic mucosa. Gut 1983;24:1176-82.

7. Sharon P, Stenson WF. Enhanced synthesis of leukotriene $\mathrm{B}_{4}$ by colonic mucosa in inflammatory bowel disease. Gastroenterology 1984;86:452-60.

8. Peskar BM, Dreyling KW, May B, et al. Increased formation of leukotriene $\mathrm{B}_{4}$ and sulphidopeptide-leukotrienes by rectal mucosa of patient with Crohn's disease and ulcerative colitis. Gut 1985;26:A542-3.

9. Lauritsen K, Laursen LS, Bukhave K, Rask-Madsen J. Effects of topical aminosalicylic acid and prednisolone on prostaglandin $\mathrm{E}_{2}$ and leukotriene $\mathrm{B}_{4}$ levels determined by equilibrium in vivo dialysis of rectum in relapsing ulcerative colitis. Gastroenterology 1986;91:837-46.

10. Lauritsen K, Laursen LS, Bukhave K, Rask-Madsen J. In vivo effects of orally administered prednisolone on prostaglandin and leukotriene production in ulcerative colitis. Gut 1987;28:1095-9.

11. Luaritsen K, Laursen LS, Bukhave K, Rask-Madsen J. In vivo profiles of eicosanoids in ulcerative colitis, Crohn's colitis, and Clostridium difficile colitis. Gastroenterol 1988;95:111-7.

12. Zipser RD, Nast CC, Lee M, Kao H, Duke $\mathrm{R}$. In vivo production of leukotriene $\mathrm{B}_{4}$ and $\mathrm{C}_{4}$ in experimental colitis. Gastroenterology 1986;90:1705.

13. Sharon P, Stenson WF. Metabolism of arachidonic acid in acetic acid colitis in rats: Similarity to human inflammatory bowel disease. Gastroenterology 1985;88:55-63.

14. Stenson WF. Role of Lipoxygenase Products in Inflammatory Bowel Disease. In: Rachmilewitz D, ed. Inflammatory Bowel Disease. The Hague: Martinus Nijhoff 1986.

15. Boughton-Smith NK, Wallace JL, Morris GP, Whittle BJR. The effect of anti-inflammatory drugs on eicosanoid formation in a chronic model of inflammatory bowel disease in the rat. Br J Pharmacol 1988;94:65-72.

16. Wallace JL, MacNaughton WK, Morris GP, Beck PL. Inhibition of leukotriene synthesis markedly accelerates healing in a rat model of inflammatory bowel disease. Gastroenterology 1989;96:29-36.

17. Allgayer H, DeSchryver K,
Stenson W. Treatment with 16,16'. dimethylprostaglandin $\mathrm{E}_{2}$ before and after induction of colitis with trinitrobenzenesulfonic acid in rats decreases inflammation.

Gastroenterology 1989;96:1290-300.

18. Stenson WF, Chang K, Williamson JR. Tissue differences in vascular permeability induced by leukotriene $B$ and prostaglandin $E_{2}$ in the rat. Prostaglandins 1986;32:5-19.

19. Musch MW, Miller RJ, Field M, Siegel M. Stimulation of colonic secretion by lipoxygenase metabolites of arachidonic acid. Science 1982;217:1255-6.

20. Saverymuttu $S H$, Peters AM, Lavender JP, Chadwick VS, Hodgson $\mathrm{HJ}$. In vivo assessment of granulocytic migration to diseased bowel in Crohn's disease. Gut 1985;26:378-83.

21. Ford-Hutchinson AW, Bray MA, Doing MV, Shipley ME, Smith JF. Leukotriene B, a potent chemotactic and aggregating substance released from polymorphonuclear leukocytes. Nature 1984;266:264-5.

22. Lobos EA, Sharon P, Stenson WF. Chemotactic activity in inflammatory bowel disease. Dig Dis Sci 1987;32:1380-8.

23. Das KM, Dubin R. Clinical pharmacokinetics of sulphasalazine. Clin Pharmacokin 1976;1:406-25.

24. MacDermott RP, Kane MG, Steele LL, Stenson WF. Inhibition of cytotoxicity by sulphasalazine. I. Sulphasalazine inhibits spontaneous cell-mediated cytotoxicity by control and inflammatory bowel disease peripheral blood and intestinal mononuclear cells. Immunopharm 1986;11:101-10. 


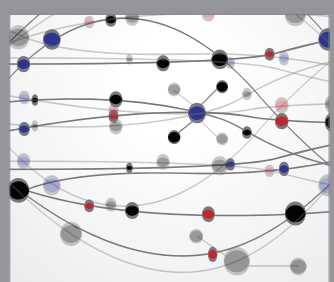

The Scientific World Journal
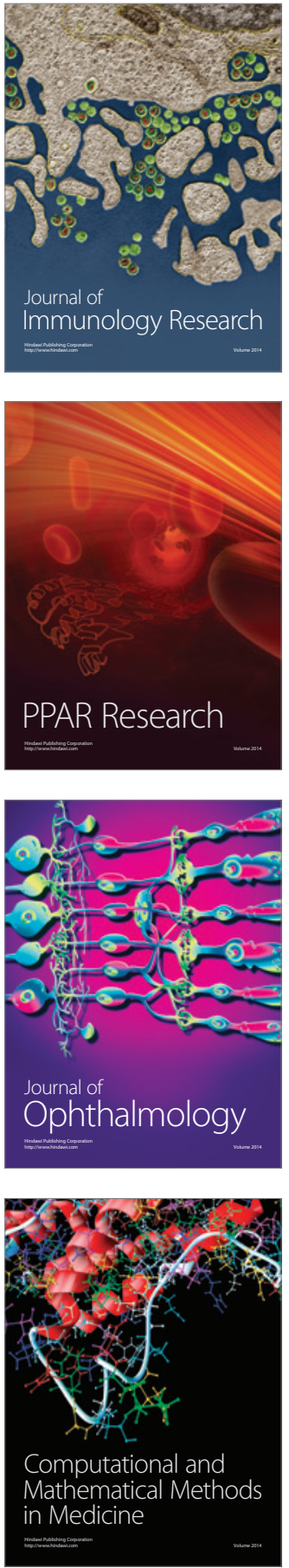

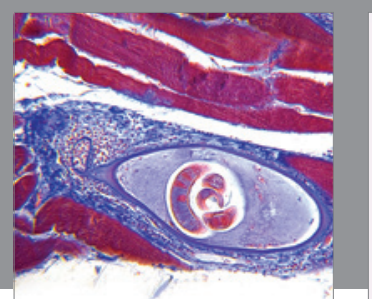

Gastroenterology Research and Practice

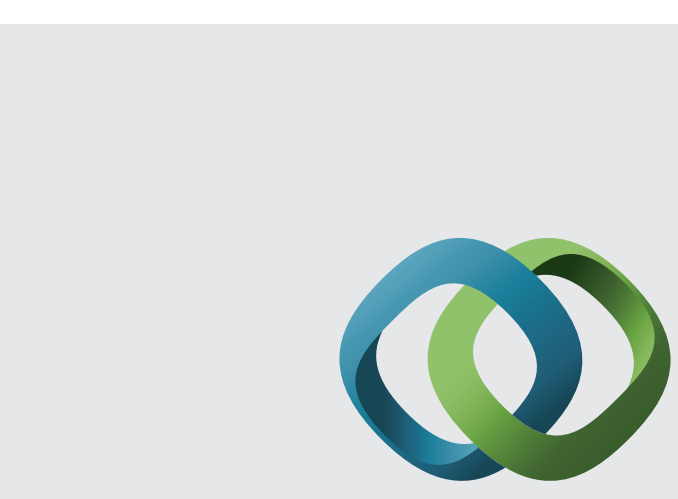

\section{Hindawi}

Submit your manuscripts at

http://www.hindawi.com
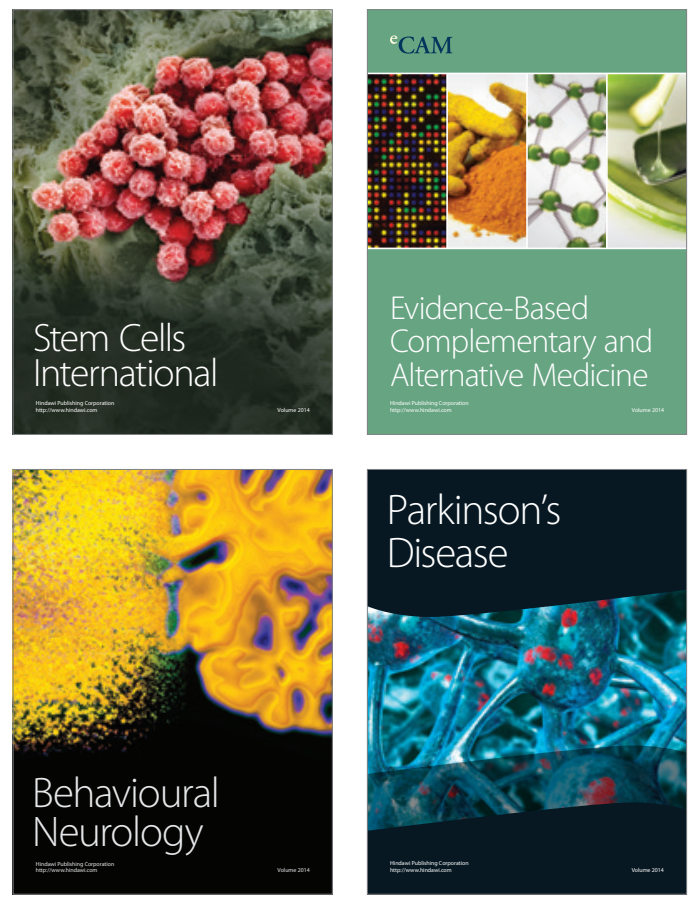
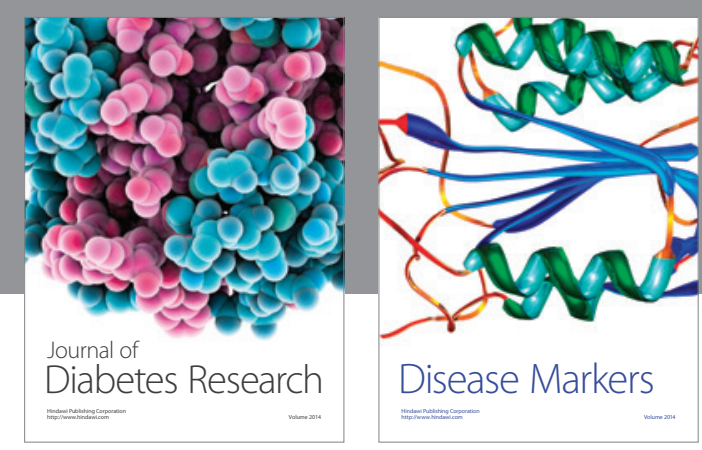

Disease Markers
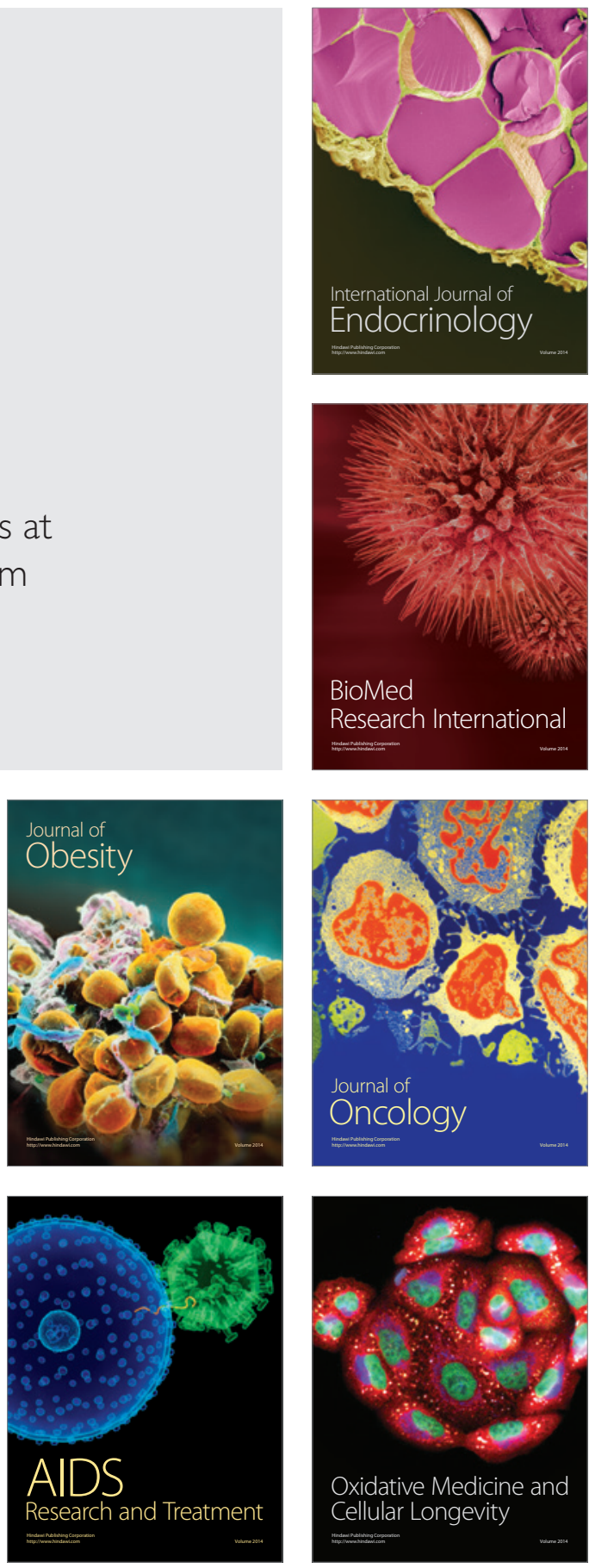